\title{
Genetic basis of hepatitis virus-associated hepatocellular carcinoma: linkage between infection, inflammation, and tumorigenesis
}

\author{
Haruhiko Takeda $^{1} \cdot$ Atsushi Takai $^{1} \cdot$ Tadashi Inuzuka $^{1} \cdot$ Hiroyuki Marusawa $^{1}$
}

Received: 20 September 2016/ Accepted: 23 September 2016/Published online: 6 October 2016

(C) Japanese Society of Gastroenterology 2016

\begin{abstract}
Hepatitis virus infection is a leading cause of chronic liver disease, including cirrhosis and hepatocellular carcinoma (HCC). Although anti-viral therapies against hepatitis $\mathrm{B}$ virus (HBV) and hepatitis $\mathrm{C}$ virus (HCV) have dramatically progressed during the past decade, the estimated number of people chronically infected with HBV and/or HCV is $\sim 370$ million, and hepatitis virus-associated hepatocarcinogenesis is a serious health concern worldwide. Understanding the mechanism of virus-associated carcinogenesis is crucial toward both treatment and prevention, and the recently developed whole genome/exome sequencing analysis using next-generation sequencing technologies has contributed to unveiling the landscape of genetic and epigenetic aberrations in not only tumor tissues but also the background liver tissues underlying chronic liver damage caused by hepatitis virus infection. Several major mechanisms underlie the genetic and epigenetic aberrations in the hepatitis virus-infected liver, such as the generation of reactive oxidative stress, ectopic expression of DNA mutator enzymes, and dysfunction of the DNA repair system. In addition, direct oncogenic effects of hepatitis virus, represented by the integration of HBVDNA, are observed in infected hepatocytes. Elucidating the whole picture of genetic and epigenetic alterations, as well as the mechanisms of tumorigenesis, will facilitate the development of efficient treatment and prevention strategies for hepatitis virus-associated HCC.
\end{abstract}

Hiroyuki Marusawa

maru@kuhp.kyoto-u.ac.jp

1 Department of Gastroenterology and Hepatology, Graduate School of Medicine, Kyoto University, 54 Kawahara-cho, Shogoin, Sakyo-ku, Kyoto 606-8507, Japan
Keywords Hepatocarcinogenesis · Cirrhosis · Inflammation · Next-generation sequencing · Whole genome sequencing

$\begin{array}{ll}\text { Abbreviations } \\ \text { AAV2 } & \text { Adeno-associated virus 2 } \\ \text { AFP } & \text { Alfa-fetoprotein } \\ \text { AID } & \text { Activation-induced cytidine deaminase } \\ \text { APOBEC } & \text { Apolipoprotein B editing complex } \\ \text { DAA } & \text { Direct-acting antiviral } \\ \text { DN } & \text { Dysplastic nodule } \\ \text { HBV } & \text { Hepatitis B virus } \\ \text { HCC } & \text { Hepatocellular carcinoma } \\ \text { HCV } & \text { Hepatitis C virus } \\ \text { IFN } & \text { Interferon } \\ \text { LINE } & \text { Long interspersed nuclear element } \\ \text { NA } & \text { Nucleotide analogue } \\ \text { NASH } & \text { Nonalcoholic steatohepatitis } \\ \text { NGS } & \text { Next-generation sequencing } \\ \text { RFA } & \text { Radiofrequency ablation } \\ \text { ROS } & \text { Reactive oxygen species } \\ \text { STV } & \text { Structural variations } \\ \text { SVR } & \text { Sustained virological response } \\ \text { TACE } & \text { Transarterial chemoembolization }\end{array}$

\section{Introduction}

Hepatocellular carcinoma (HCC) is one of the most unfavorable malignancies worldwide. It is currently the second leading cause of cancer-related deaths, with an estimated 500,000-600,000 deaths/year [1-3]. To date, multidisciplinary strategies for HCC treatment include surgery, 
radiofrequency ablation, transarterial chemoembolization, and molecular targeting therapy [4-9]. The multi-centric manner of tumorigenesis that is characteristic of $\mathrm{HCC}$ development, however, makes it extremely difficult to achieve curative therapy for HCC. Indeed, most HCC patients experience several cancer recurrences, even after local curative treatment by either surgical resection or radiofrequency ablation $[4,10,11]$. As a result, the overall survival rate is very poor in patients with multiple HCCs $[5,12,13]$.

HCC has a diverse etiology; viral infection with hepatitis B virus (HBV) or hepatitis C virus (HCV), alcohol intake, non-alcoholic steatohepatitis, or aflatoxin. Among them, viral hepatitis is the major cause of HCC in Japan and East Asian countries [2]. HCC frequently develops in the setting of chronic hepatitis and/or cirrhosis following HCV and/or HBV chronic infection. The development of anti-viral therapies against hepatitis viruses has rapidly progressed during the past decade. Suppression and/or eradication of hepatitis virus could contribute to reduce the incidence of HCC, but multicentric tumors often develop in patients after clearance of the infecting viruses [14, 15]. Thus, virus-associated hepatocarcinogenesis continues to be a serious matter, and elucidation of the mechanism of virus-associated carcinogenesis in the liver is urgently needed.

Genetic aberrations including nucleotide alterations and structural variations (STVs), as well as epigenetic changes, including DNA methylations and histone modifications, are accumulated in cancer cells [16-18]. The accumulation of several genetic alterations is essential for the transformation of normal cells into cancer cells, and epigenetic changes provide additional malignant potential. The recent development of the next-generation sequencing technologies provides novel strategies for analyzing the human genome and epigenome. Use of these new technologies has gradually begun to unveil the landscape of genetic and epigenetic aberrations of a variety of human tumors, including HCC [19-30]. In this article, we review the recent findings concerning genetic and epigenetic aberrations that accumulate during the development of hepatitis virus-associated HCC.

\section{Trends in HCV-related liver disease; possibility of HCC development after viral eradication}

More than 130 million people are estimated to be infected with HCV worldwide [31]. In the natural course of persistent viral infection, approximately $20-30 \%$ of the patients develop liver cirrhosis within 20-30 years after $\mathrm{HCV}$ infection [32], and the estimated incidence of HCC in patients with $\mathrm{HCV}$-associated liver cirrhosis is $1-8 \%$ per year [33-35]. A number of studies reported the risk factors of HCV-related HCC development, including the level of fibrosis/cirrhosis present at diagnosis, alfa-fetoprotein levels, alanine aminotransferase levels, sex, age, platelet count, and HCV RNA levels [36-38]. Multicentric tumorigenesis, a major characteristic of $\mathrm{HCV}$-associated HCC, makes curative treatment for HCC difficult, leading to high mortality.

Conventional anti-HCV therapy is based on treatment regimens that include interferon (IFN), but the sustained virological response (SVR) rate is insufficient, and the indications of IFN-based therapy are limited due to adverse effects [39]. Since 2011, new drugs targeting HCV proteins, so-called direct-acting antivirals (DAAs), have been introduced to clinical practice, which have dramatically improved the SVR rate [40-43]. To date, IFN-free regimens with DAAs have become the standard anti-HCV therapy, and the current regimens can eradicate $\mathrm{HCV}$ with a success rate greater than $95 \%$ [44-48].

Although several clinical studies have demonstrated that eradication of HCV with IFN-based regimens results in the suppression of liver carcinogenesis [49-51], HCC can still develop after viral eradication. Indeed, the 5-year HCC incidence after SVR by IFN-based antiviral therapy for HCV-related chronic liver disease is $2.3-8.8 \%$ [52]. Although the cancer incidence appears to be lower in SVR patients than in patients that do not receive anti-HCV therapy, these results indicate that eradication of HCV by IFN-based therapy does not completely suppress liver carcinogenesis in patients with chronic HCV infection [53]. On the other hand, DAAs eradicate HCV through very different mechanisms compared with the IFN-based regimen, and it has not been clarified whether clearance of HCV by DAAs can fully suppress the development of HCC. Of note, more than $20 \%$ of HCV-positive patients who received DAA treatment after curative therapy for local HCC experienced HCC recurrence [54]. Thus, the efficacy of $\mathrm{HCV}$ eradication by DAA regimens for the prevention of HCC remains unclear, and accumulating evidence suggests that a subset of patients receiving DAA therapy, especially older people and those with cirrhosis, could develop HCC after achieving SVR [55], which would be one of the most significant problems in HCVrelated hepatocarcinogenesis in the coming decade.

\section{Trends in HBV-related liver disease; persistent viral infection and HCC development}

An estimated 240 million people are chronically infected with HBV worldwide, and HBV prevalence is highest in sub-Saharan Africa and East Asia, where between 5 and $10 \%$ of the adult population is chronically infected with 
$\mathrm{HBV}$ [56]. HBV infection is a main risk factor for $\mathrm{HCC}$ development in the Asian-Pacific region and Africa, and recent estimates attribute over $50 \%$ of HCC cases worldwide to HBV infection $[2,57]$. Noteworthy is that HBVpositive patients develop HCCs at a relatively younger age compared with HCC cases related to alcohol, nonalcoholic steatohepatitis (NASH), and HCV. In addition, up to onethird of HBV-related HCC patients develop tumors without cirrhosis [57, 58], and even carriers of inactive HBV whose serum alanine aminotransferase levels are within the normal range are also at substantial risk for HCC compared with individuals without HBV infection [59]. Moreover, high serum HBV DNA level is a strong risk predictor of HCC [60]. These findings suggest that HBV infection itself has oncogenic potential and HCC could develop in HBVinfected liver lacking active inflammation and the resultant liver damage and/or fibrosis.

Anti-HBV treatment is based on pegylated-IFN and nucleotide analogues such as entecavir and tenofovir, both of which efficiently suppress HBV replication [61-66]. Several studies demonstrated the efficacy of anti-HBV therapies for suppressing the incidence of HCC. For example, IFN therapy inhibits the development of HBVrelated $\mathrm{HCC}$ and extends survival time. One study reported that the cumulative incidence of HCC development is significantly lower in patients treated with IFN than in those who are not treated (1.5 vs. $11.8 \%$ during a 11.5year follow-up period), and the cumulative survival rate through the 10th year was $98 \%$ in patients treated with IFN in contrast to $57 \%$ in those not treated with IFN [67]. Nucleotide analogues also suppress the viral load, resulting in decreased cancer incidence. For example, HCC developed in $3.9 \%$ of patients receiving lamivudine and $7.4 \%$ of those with placebo treatment during a median period of 32.4 months (hazard ratio, 0.49) [64]. In addition, nucleotide analogues are effective for decreasing HCC recurrence after curative therapy against the primary tumor [68]. These findings suggest that suppressing HBV replication by anti-HBV therapies is basically effective for reducing the cancer incidence, but it is important to note that antiHBV therapy cannot achieve complete eradication of viruses from infected individuals.

HBV infection persists in the liver even after the disappearance of hepatitis B surface antigens (HBsAg) in individuals with previous exposure to the virus, retaining the serologic footprint of anti-HBc positivity, with such a status defined as occult HBV infection. Occult HBV carriers can develop HBV reactivation and liver dysfunction under certain immunosuppressive conditions $[69,70]$. The formation of covalently closed circular DNA is considered due to the persistent HBV infection in hepatocytes in individuals after seroconversion from HBsAg to anti-HBs [71]. That is, occult HBV infection could be maintained as an episomal form in the liver tissues of patients even after circulating HBV-DNA becomes undetectable following anti-HBV therapies. In addition to the latent continuous infection of HBV in an episomal form, the HBV genome could also be frequently integrated into the host genome, thereby preventing its eradication by anti-HBV therapies. Due to these specific features of HBV infection, HCC could develop in patients negative for serum HBV-DNA and/or HBsAg, irrespective of the efficacy of anti-viral therapies.

\section{Genetics of hepatitis virus-related HCC}

Represented by the novel achievements of the International Cancer Genome Consortium (ICGC), which coordinates large-scale cancer genome studies of various human cancers that are of clinical and societal importance across the globe [72], vast amounts of information concerning genetic aberrations in tumor tissues has been accumulated worldwide [19-30, 73, 74]. Whole genome sequencing analyses revealed more than 9000 point mutations per human HCC sample [73], and somatic mutations are detected in approximately $40-80$ protein-coding genes in HCC $[19,28,75]$. Mutations in the telomerase reverse transcriptase (TERT) promoter are the most prevalent in hepatitis virus-related HCC. In particular, more than $60 \%$ of HCV-related HCCs possess TERT promoter mutations $[25,76]$. These mutations might lead to telomerase reactivation, allowing cells to avoid death and acquire malignant potential. Whole genome/exome analysis demonstrated that TP53 and CTNNB1 are the most frequently mutated coding genes, and chromatin modulators, including ARIDIA and ARID2, are also recurrently mutated in HCC [24, 25, 73]. In addition, somatic mutations are detectable in a variety of genes with various oncogenic pathways, including telomere maintenance, Wnt signaling, p53/cell cycle, oxidative stress, epigenetic regulator, PI3 K-AKT-mTOR, MAPK, JAK/STAT, and hepatic differentiation [25]. These findings suggest that HCC is not caused by one particular driver mutation, but involves several carcinogenic pathways, making HCC extremely heterogeneous [19, 24, 77]. Interestingly, correlations between mutations of two different genes and/or pathways have also been examined in HCC tissues. For example, TERT promoter mutations tend to co-occur with aberrations of the Wnt signaling pathway, including CTNNB1 mutation [19], while genetic alterations in CTNNB1 and AXIN1 are mutually exclusive [28]. Analysis of this genome sequencing information in association with the clinical course of each HCC patient revealed that HCC patients with TERT promoter mutations or TP53 mutations have significantly poorer survival $[73,74]$. 
Recent whole genome analyses involving a vast number of HCC patients enabled the exhaustive detection of genetic alterations in not only coding regions, but also noncoding regions. While the most well known alterations in non-coding regions are TERT promoter mutations, a recent study revealed alterations in promoters of other genes, including TFPI2. They also showed mutations in several long intergenic noncoding RNA, including NEATI and MALAT1, and untranslated regions of chromosomes [73].

On the other hand, several studies elucidated the landscape of mutation signatures observed in various human cancers. While $\mathrm{C}>\mathrm{T}$ changes are prevalent in various sorts of gastrointestinal tumors [78], HCC due to chronic $\mathrm{HCV}$ infection frequently exhibit $\mathrm{T}>\mathrm{C}$ changes along with $\mathrm{C}>\mathrm{T}$ changes [19]. A recent Japanese study of 268 HCCs, including 159 HCV-related and 82 HBV-related HCCs, determined seven characteristic mutational signatures suggesting that background factors, such as aging, smoking, or alcohol intake, correlate with particular mutation signatures. In addition, some major genetic alterations are related to specific mutational patterns. For example, significant correlations were demonstrated between $\mathrm{T}>\mathrm{C}$ changes and TERT promoter mutation, $\mathrm{C}>\mathrm{T}$ changes and mutations in ARID family genes, and $\mathrm{C}>\mathrm{A}$ changes and TP53 mutations [73].

Not only single nucleotide variants but also STVs of chromosomes are detected in hepatitis virus-related HCC tissues. STVs are various structural alterations of chromosomes, including translocation, deletion, and tandem duplication. Broad genomic gains at 1q, 5p, 6p, 8q, 17q, 20q, and $\mathrm{Xq}$, as well as deletions at 1p, 4p-q, 6q, 8p, 13p-q, 16p-q, 17p, $21 \mathrm{p}-\mathrm{q}$, and $22 \mathrm{q}$ have been identified in HCC $[19,21,28,79,80]$. These changes cause focal amplifications in cancer-related genes such as VEGFA and FGF3/4/19/ CCND1. Interestingly, the amplification of these genes is reported to be associated with a good response to the multikinase inhibitor sorafenib [81, 82]. On the other hand, STVs affect the expression of cancer-related genes [73]. For example, expression of the tumor suppressor gene $A P C$ is decreased by translocation, deletion, or inversion of chromosomes. Not only point mutations but also STV breakpoints are detected in well-known driver genes, including TERT, ARIDIA, ARID2, and PTEN, in HCC tissues [73]. These studies suggest that STVs contribute to liver carcinogenesis by increasing the expression of oncogenes and/ or decreasing the expression of tumor suppressor genes.

\section{Integration of the viral genome}

Integration of the HBV genome into host genomic DNA is thought to be involved in the development of HBV-related HCC [57]. HBV integration is considered to be an early event in HBV infection, and might give hepatocytes a growth advantage relative to the clonal cell population. Recently, whole genome sequencing revealed that the HBV genome is integrated in approximately $80 \%$ of HBV-related HCCs [73]. On average, 2.5 HBV integration sites per HBV-positive HCC sample are identified by whole genome sequencing, and the HBx gene is the most prevalent among the integrated HBV genes [73].

HBV integration sites in the host genome might be crucial for dysregulating cell homeostasis. The evidence that the HBV genome is inserted into human genes was first demonstrated by the detection of chimera genome sequences formed by the HBV genome and RARB (retinoic acid receptor b) or $C C N A$ genes [83, 84], and thereafter other genes were identified as target genes affected by the integration of the HBV genome. Among various integration sites, HBV integrations are reported to occur most prevalently at the promoter and gene body of the TERT gene $(18-22 \%)$. HBV also recurrently integrates into the following cancer-related genes: MLL4, CCNE1, SENP5, ROCK1, or SOX2 [73, 74, 85]. HBV integration does not promote HBV replication, while integration into the gene body or promoter region of a cancer-related gene could lead to both altered expression of the target gene and genomic DNA instability. For example, HBV integration into the TERT promoter conspicuously increases TERT expression compared with point mutations of the TERT promoter region [73]. Interestingly, HBV integration into the TERT promoter is mutually exclusive with point mutations and STVs of TERT in HBV-related HCC [73]. In addition to the protein-coding genes, recurrent HBV integration sites are also detected within or near repetitive, non-coding sequences, such as long interspersed nuclear elements (LINEs), Alu (named after the restriction enzyme specifically cutting those sequences), other short interspersed nuclear elements, and the long terminal repeats of endogenous retroviruses. HBV integration into the LINE1 sequence results in the generation of an HBx-LINE1 chimeric transcript, which was detected in 21 of $90(23 \%)$ tumors of HBV-related HCC patients and is significantly associated with poor survival of HCC patients [86].

Recently, an integrated viral genome other than HBV was newly identified in tumor samples from HCC patients. In 2015, Nault et al. first reported that the adeno-associated virus 2 (AAV2) genome was integrated into the host genome in $\sim 5 \%$ HCC samples [87]. AAV2 is a defective DNA virus that does not possess replicative ability by itself and is integrated into the human genome in a quiescent state, suggesting that the integrated viral genome itself might be non-pathogenic [88]. Interestingly, AAV2 integration was detected in TERT, KMTB2, CCNA2, and $C C N E 1$ - the same genes affected by HBV integration, and also in other cancer-related genes, including TNFSF 10 
coding TRAIL (TNF-related apoptosis-inducing ligand). The expression levels of several cancer-related genes in which AAV2 integration occurred are significantly elevated in HCC tissues. Fujimoto et al. also demonstrated AAV2 genome integrations in three HCC samples by analyzing whole-genome sequencing reads unmapped to the human genome [73]. All three samples were hepatitis virus-associated $\mathrm{HCCs}$ and the integration sites were detected in introns of $K M T 2 B$ and $C C N E 1$, and an intergenic region on chromosome 5. KMT2B is a chromatin regulator and $C C N E 1$ is associated with cell cycle regulation. The coincidence of HBV integration and AAV2 integration was observed in one HCC sample in which elevated expression of $K M T 2 B$ was detected [73]. Although AAV2 is used for gene introduction because of its nonpathogenic nature, these recent findings suggest that AAV2 is a new candidate pathogenic virus related to human HCC, in addition to HBV and HCV [89].

\section{Epigenetics of hepatitis virus-related HCC}

DNA modifications, such as DNA methylations and histone modifications, could control the expression of genome information without nucleotide changes [17]. Representative alterations of DNA methylation associated with carcinogenesis include focal hypermethylations of $\mathrm{CpG}$ islands of the tumor suppressor genes, leading to their inactivation, and genome-wide hypomethylation, resulting in genome instability. Various alterations in DNA methylation, focal hypermethylation of specific genes, and global hypomethylation of genomic DNA, are observed in hepatitis virus-related HCC and could affect the expression level of tumor-related genes [90]. Commonly hypermethylated genes in human HCC include RASSF1A, p15, p16, SOCS1, SOCS3, and RB1, which are involved in important biologic processes, such as cell cycle regulation, apoptosis, and cell growth [91]. Shen et al. analyzed the pathways of hypermethylated or hypomethylated genes using 62 pairs of human HCC tumors and adjacent non-tumor tissues, and reported that expression levels of tumor-related genes belonging to $\mathrm{G}$ protein, phosphatidylinositol-3-kinase, interleukin, insulin-like growth factor, and Wnt signaling pathways are affected by DNA methylation [92]. Tao et al. analyzed the DNA methylation status in single hepatocytes isolated from the tumor tissue of HBV-related HCC patients, and demonstrated that DNA methylations induce abnormalities in cell function, including cell adhesion and apoptosis [93].

Interestingly, genomic analysis identified various genetic alterations of epigenetic modifiers in human cancers, including DNA methyltransferases (DNMTs) regulating DNA methylation, EZH2 coding histone methyltransferase of $\mathrm{H} 3 \mathrm{~K} 27$, and SNF5 participating in chromatin remodeling
$[16,94] . \mathrm{HCC}$ tissues contain mutations of genes essential for maintaining the chromatin structure, including ARIDIA, ARIDIB, ARID2, and MLL4 [25]. Mutations of these epigenetic modifiers lead to profound epigenetic changes, including aberrant DNA methylation, histone modifications, and nucleosome positioning [16], resulting in abnormal gene expression and genomic instability, which may predispose to HCC development.

DNA methylations correlate with the transcription of not only protein-coding genes, but also non-coding RNA, such as microRNA and long noncoding RNA. MicroRNAs are short noncoding RNA molecules that post-transcriptionally repress gene expression, and a number of microRNAs are reported to correlate with cancer development through modifications of gene expression [95]. A recent study demonstrated that abnormal hypermethylation suppresses the transcription of various microRNAs, followed by dysfunction of intracellular signaling pathways. For example, abnormal methylation of the HOXB4 gene leads to the inactivation of miR-10a, resulting in the activation of the $\mathrm{NF \kappa B}$ signaling pathway in human HCC tumor tissues [96]. In addition, methylation-mediated repression of several microRNAs correlates with tumor aggressiveness of human HCC [97-99].

On the other hand, hypomethylation specific to cancer tissue is observed in some genes [100]. To date, several hypomethylated genes have been detected in HCC [91], while mega-scaled hypomethylated $\mathrm{CpG}$ islands exist in nearby telomeres and centromeres of chromosomes [100]. In addition, LINE-1, a retrotransposon, is hypomethylated in various types of cancer, and is considered to play an important role in carcinogenesis [101]. In HCC, global DNA demethylation is also detected in LINE-1 and other repeated genome sequences, including IAP, Alu, and SAT, contributing to the putative enhanced instability of genomic DNA [102].

Interestingly, methylation status differs between HBVand HCV-related HCCs [90, 103, 104]. Several genes, including $H O X 9, R A S S F 1$, and SFRP1, are methylated more frequently in $\mathrm{HBV}$-positive $\mathrm{HCC}$, while methylation of $C D K N 2 A$ is highly prevalent in $\mathrm{HCV}$-positive $\mathrm{HCC}$ [103]. Methylation analysis of gene promoter regions revealed that the methylation of 15 cancer-related genes belonging to RAS/RAF/ERK or $\mathrm{Wnt} / \beta$ catenin pathways are specific to HCV-positive HCC patients [105].

\section{Genetic alterations accumulated in hepatitis virus- infected liver}

Inflammation-associated cancers include not only hepatitis virus-associated HCC, but also gastric cancer arising from Helicobacter pylori-associated atrophic gastritis and colitic 
cancer arising from inflammatory bowel disease. A common characteristic of these inflammation-associated cancers is multicentric tumorigenesis, suggesting that the genetic aberrations required for malignant transformation widely accumulate in noncancerous inflamed tissues $[18,106]$. Consistently, several studies have demonstrated various mechanisms underlying the enhanced genetic instability caused by hepatitis virus infection and the resultant inflammatory response (Fig. 1).

Oxidative stress mediates genetic aberrations in inflamed tissues. Reactive oxygen species (ROS) and reactive nitrogen species are considered potential genotoxic factors [107]. Reactive oxygen and nitrogen species induce various types of DNA damage, including point mutations, DNA adducts, and single- or double-stranded DNA breaks [108, 109]. One of the main DNA adducts, 8-oxo-2'-deoxyguanosine, formed by oxidative stress could be involved in DNA damage [107]. As hepatocytes carry out many metabolic reactions, ROS are generated on a routine basis in the liver [110-112]. ROS generation is increased in patients with chronic liver diseases, including HCV-related hepatitis [113, 114]. A viral protein produced by HCV increases the expression of ROS [115-118]. In addition, HCV infection inhibits DNA repair through the production of ROS and reactive nitrogen species by interfering with the ATM-NBS1/Mre11/Rad50 DNA repair pathway in hepatocytes [119].

Nucleotide editing enzymes, the apolipoprotein B editing complex (APOBEC) family of proteins, are suggested to be involved in the induction of genetic alterations in various human malignancies. Among APOBEC family proteins, activation-induced cytidine deaminase (AID) plays a role as a DNA mutator enzyme and is associated with inflammation-related carcinogenesis [106, 120-122]. Under physiologic conditions, AID is ordinarily expressed in activated B-lymphocytes, and is required for somatic hypermutation and class-switch recombination of immunoglobulin genes [123, 124]. AID is induced in human hepatic, gastric, and biliary epithelial cells, however, in response to pro-inflammatory cytokines and or pathogen infections such as HCV [125]. Consistently, AID expression is observed in hepatocytes of chronically inflamed HCV-infected liver, while AID expression is not observed in normal liver tissues [126, 127]. In vivo studies revealed that constitutive AID expression promotes tumorigenesis by enhancing the susceptibility to mutagenesis in a variety of epithelial organs, including the liver $[125,128,129]$. These findings suggest that chronic inflammation caused by HCV infection triggers the aberrant upregulation of AID in hepatocytes, leading to the genomic instability required for tumorigenesis.

The high-fidelity DNA repair system has an important role in preventing the generation of genetic abnormalities, and DNA repair function abnormalities are involved in inflammation-associated carcinogenesis. The DNA repair system plays a critical role in the removal of damaged or incorrect bases and DNA resynthesis by DNA polymerase [130]. Among them, mismatch repair is an excision repair process that removes mismatched bases, protecting the genome against mutagenic activity derived from both intrinsic and extrinsic factors $[130,131]$. Dysfunction of the DNA repair system, however, can trigger the emergence of somatic mutations, resulting in enhanced genetic instability. In fact, several types of congenital and acquired human cancers contain mutations or exhibit methylated silencing of DNA repair genes, including $M L H 1$ and polymerase $\varepsilon$ (POLE) [132, 133]. We recently demonstrated that the expression level of $M S H 2$, a representative mismatch repair protein, is downregulated by tumor necrosis factor- $\alpha$ in inflamed hepatocytes [134]. In addition, hepatocyte-specific defects of $M S H 2$ result in the development of liver tumors with the histologic features of HCC. HCV infection also induces error prone polymerases that can contribute to enhance susceptibility to mutagenesis [135]. Therefore, in addition to genotoxic factors, dysfunction of the DNA repair system can contribute to the induction of genetic aberrations during hepatitis-associated tumorigenesis.

On the other hand, several previous studies revealed direct effects of HBV viral proteins on the enhanced susceptibility of genetic alterations. For instance, an in vitro study demonstrated that HBx protein induces centrosome abnormalities, leading to an increased frequency of defective mitoses and chromosome transmission errors [136]. HBx also inactivates TP53, collapses the mitotic checkpoint, and interacts with the DNA repair protein DDB1 to induce genetic instability [137]. Together, chronic inflammation caused by viral infection, direct oncogenic effects of the viral protein itself, and integrated HBV-DNA could contribute to the accumulation of genetic aberrations in hepatitis virus-infected liver.

Consistent with the presumed enhanced genetic instability of the liver underlying hepatitis virus infection in in vitro and in vivo models, genetic aberrations are detected in human cirrhotic liver tissues with hepatitis virus infection. We performed whole exome sequencing of HCV-positive cirrhotic liver tissues and elucidated the landscape of somatic mutations that latently accumulate in the liver following chronic $\mathrm{HCV}$ infection [138]. A number of somatic mutations are detectable in cirrhotic liver tissues, and the mutation signatures detected in cirrhotic liver are similar to those observed in HCC tissues. Importantly, mutations of cancer-related genes, including TP53 and CTNNB1, are detected in cirrhotic tissues, while variant allele frequencies are lower than those of tumor tissues. These 
Fig. 1 Mechanisms of hepatitis virus-associated hepatocarcinogenesis. HCV or HBV infection causes chronic inflammation in the liver tissue, leading to ROS generation, elevated expression of DNA mutator, and dysfunction of DNA repair function. Direct effects of hepatitis virus, including the oncogenic effects of HBx protein and HBV genome integration, can also contribute to enhance genomic instability. In addition, AAV2 genome integration into host genome could be associated with dysregulation of some cancer-related genes. These multiple factors coordinately induce the accumulation of genetic and epigenetic alterations in liver tissue underlying chronic hepatitis or cirrhosis, leading to the development of HCC

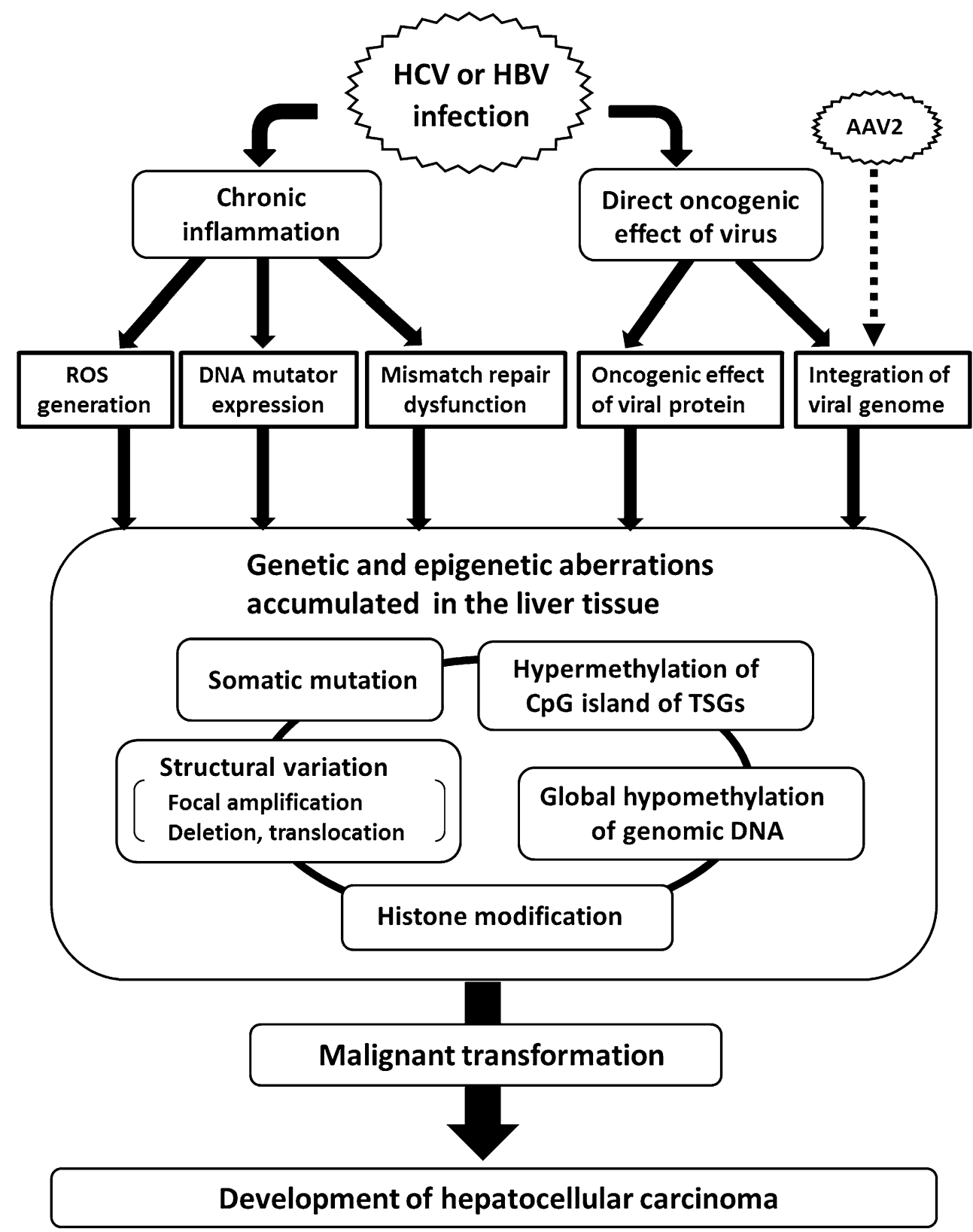

Development of hepatocellular carcinoma findings suggest that $\mathrm{HCV}$-infected cirrhotic liver tissues possess various genetic aberrations, providing the putative basis of tumorigenesis in cirrhotic $\mathrm{HCV}$-infected liver [138]. Similarly, genetic alterations are already detectable in HBV-positive cirrhosis liver during the early phase of hepatocarcinogenesis. Nault et al. analyzed the genetic alterations of dysplastic nodules (DNs), pre-neoplastic lesions in liver cirrhosis infected with $\mathrm{HBV}$, and demonstrated that TERT promoter mutations are detected in DNs and additional mutations in cancerrelated genes including TP53 and CTNNB1 are gained along with disease progression from early $\mathrm{HCC}$ to progressive HCC [139].

\section{Epigenetic alterations accumulated in hepatitis} virus-infected liver

Changes in the DNA methylation status are considered to be an early event in hepatocarcinogenesis [140]. Similar to genetic aberrations, epigenetic aberrations, including DNA hypomethylation and/or promoter gene CpG hypermethylation, are observed in the noncancerous liver tissues, such as cirrhotic nodules or DNs [141-143]. Although the mechanisms of the generation of epigenetic aberrations are not fully understood, epigenetic aberrations are caused by inflammation-related processes as well as direct effects of the hepatitis viral proteins. 
Fig. 2 Tumor development in the liver after eradication or suppression of hepatitis viruses. Genetic and/or epigenetic aberrations might accumulate in the background in the liver along with long-term hepatitis virus infection. Although antiviral therapies eradicate $\mathrm{HCV}$ or suppress HBV, these genetic and/or epigenetic aberrations that accumulate in chronically damaged liver tissue could persist unchanged and provide the basis of HCC development after anti-viral therapy

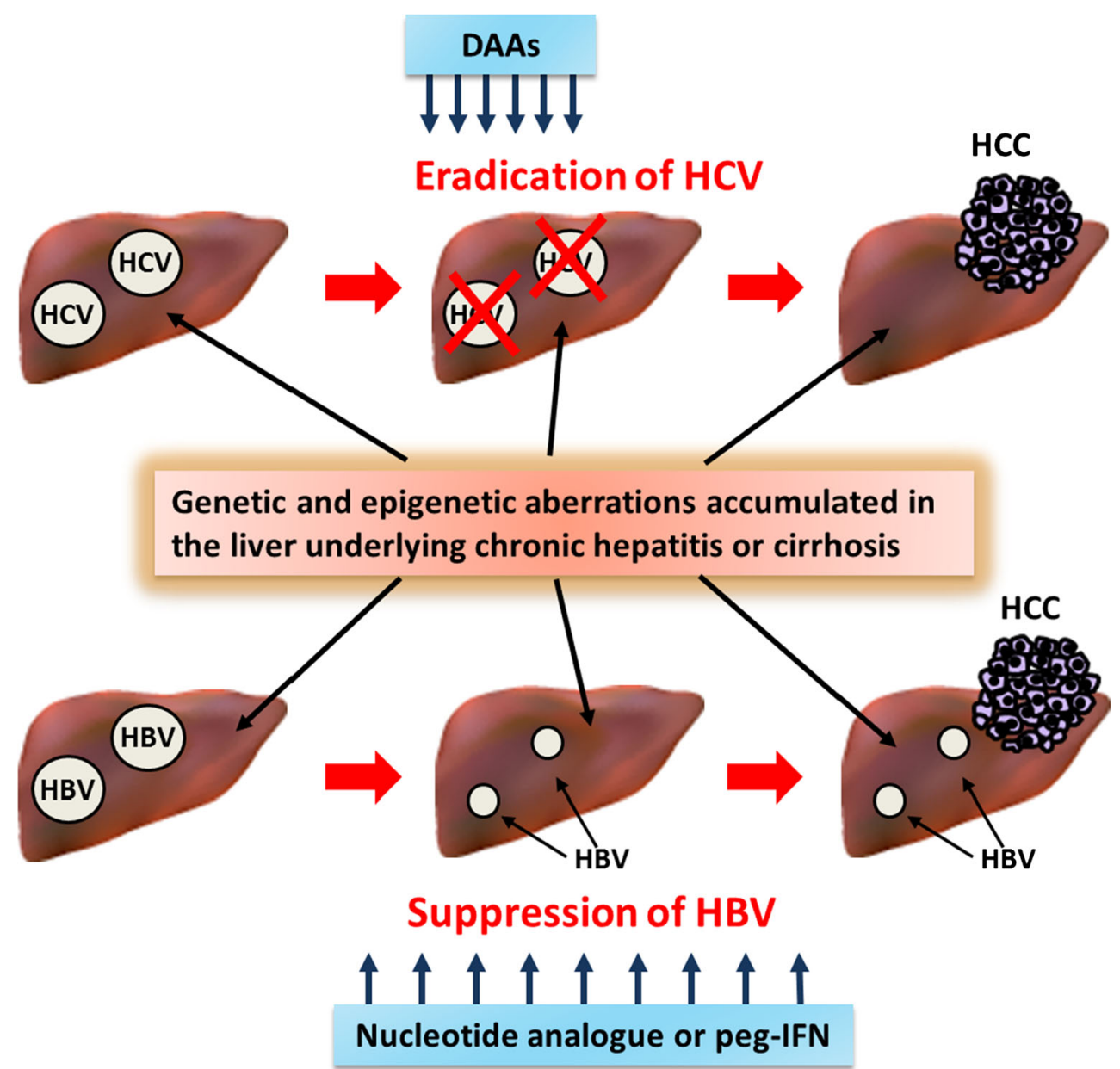

Chronic inflammation by viral infection disrupts the DNA methylation status through the immune response as well as by the induction of ROS. HBV or HCV infection induces genome-wide, time-dependent changes in DNA methylation in infected mice with humanized livers. Inhibition of natural killer cells or administration of a neutralizing IFN- $\gamma$ antibody inhibits methylation changes, suggesting that natural killer cell function is a key player in inducing aberrant DNA methylation in human hepatocytes after initial HBV and HCV infection [144]. ROS induced by chronic HCV infection could alter histone modification to the repressive form at $\mathrm{CpG}$ island-containing tumor suppressor gene promoters, leading to tumor suppressor gene inactivation [145]. These findings suggest that chronic inflammation associated with hepatitis virus infection might cause epigenetic alterations through the immune response as well as through the induction of oxidative stress.

A direct effect of the viral protein is also reported to contribute to the emergence of epigenetic aberrations in the liver with hepatitis virus infection. For example, HBx protein directly binds the chromatin complex and increases the activity of DNMT through the upregulation of DNMT1 and DNMT3A1. Targeted deregulation of DNMT by HBx is considered to promote both specific regional hypermethylation and global hypomethylation in $\mathrm{HBV}$-infected HCC patients [146]. The IGFBP-3 promoter region was hypermethylated in HBx-positive noncancerous tissues as well as HCC tissues, whereas regional hypermethylation was not detected in HBx-negative noncancerous tissues. Thus, HBx protein may play a direct role in altering the DNA epigenetics in HBV-infected hepatocytes.

In clinical specimens, $\mathrm{HCV}$-infected liver tissues have a number of methylated tumor suppressor genes. Methylation of tumor suppressor gene promoters is more prominent in $\mathrm{HCV}$-positive liver tissues than in HBV-positive or hepatitis virus-negative liver tissues [90]. In addition, methylation of tumor suppressor gene promoters is more prevalent in liver cirrhosis than in chronic hepatitis [147]. These findings suggest that chronic HCV infection accelerates the methylation process. Nishida et al. classified methylation events into three patterns: methylation events showing prominent differences between non-cancerous liver and early HCC, methylation events showing a gradual increase with tumor 
progression, and methylation that is detected only in advanced tumors. Thus, the patterns of these methylation events have tremendous diversity [140]. Importantly, elevated levels of methylation detected in early $\mathrm{HCC}$ are also observed in non-cancerous liver, although the levels are much lower than those in HCCs [90]. With regard to clinical importance, the number of methylated tumor suppressor genes in the $\mathrm{HCV}$-infected liver is positively correlated with time-to-HCC occurrence [140].

Several studies evaluated the accumulation of DNA methylation during multistep carcinogenesis. Lee et al. investigated the methylation status of $\mathrm{CpG}$ islands of several genes to determine the methylation profile of multiple tumor-related genes during multistep hepatocarcinogenesis, using tissues of hepatitis virus (mainly HBV)-associated chronic hepatitis, liver cirrhosis, DNs, and HCC. The DNA methylation frequencies of the genes examined increased as the liver disease progressed [141]. Um et al. examined the methylation status of several tumor-related genes in low-grade DNs, high-grade DNs, and early HCC with $\mathrm{HBV}$ infection, and demonstrated a stepwise increase of methylating events during HBV-related multistep hepatocarcinogenesis [142]. They also suggested that epigenetic changes frequently occur in the early stages of HCC development [142].

\section{Conclusions}

Recent progress in genomic analyses, including whole genome/exome sequencing, has rapidly unveiled the landscape of genetic and epigenetic aberrations during the process of hepatitis virus-associated hepatocarcinogenesis. Noteworthy is that a variety of molecular alterations latently occur in hepatitis and/or cirrhotic liver tissue. The fact that genetic and/or epigenetic aberrations have already accumulated in the background liver along with long-term hepatitis virus infection strongly suggests that chronically damaged liver tissue possesses significant malignant potential, even after eradication or suppression of the infecting hepatitis viruses (Fig. 2). Thus, further clinical examination is necessary to determine which patients are likely to develop HCC despite anti-viral therapy, and which patients indicated for anti-viral therapy for complete prevention of HCC development. Furthermore, HCCs are caused not only by hepatitis virus infection but also other etiologies, such as steatohepatitis and alcohol intake [148]. Thus, these factors should be taken into consideration as additional risk factors that may enhance HCC development after the completion of anti-hepatitis virus therapy. All clinicians should be aware that hepatocarcinogenesis cannot be fully prevented by anti-viral therapy alone and attention should be paid to the multiple factors associated with hepatocarcinogenesis, including individual life style.

\section{Compliance with ethical standards}

Conflict of interest The authors declare that there are no potential conflicts to disclose.

\section{References}

1. Torre LA, Bray F, Siegel RL, et al. Global cancer statistics, 2012. CA Cancer J Clin. 2015;65:87-108.

2. El-Serag HB. Epidemiology of viral hepatitis and hepatocellular carcinoma. Gastroenterology. 2012;142:1264-73.

3. Ferlay J, Soerjomataram I, Dikshit R, et al. Cancer incidence and mortality worldwide: sources, methods and major patterns in GLOBOCAN 2012. Int J Cancer. 2015;136:E359-86.

4. Forner A, Llovet JM, Bruix J. Hepatocellular carcinoma. Lancet. 2012;379:1245-55.

5. Llovet JM, Ricci S, Mazzaferro V, et al. Sorafenib in advanced hepatocellular carcinoma. N Engl J Med. 2008;359:378-90.

6. Llovet JM, Villanueva A, Lachenmayer A, et al. Advances in targeted therapies for hepatocellular carcinoma in the genomic era. Nat Rev Clin Oncol. 2015;12:408-24.

7. Bruix J, Gores GJ, Mazzaferro V. Hepatocellular carcinoma: clinical frontiers and perspectives. Gut. 2014;63:844-55.

8. Kaneko S, Ikeda K, Matsuzaki Y, et al. Safety and effectiveness of sorafenib in Japanese patients with hepatocellular carcinoma in daily medical practice: interim analysis of a prospective postmarketing all-patient surveillance study. J Gastroenterol. 2016;51:1011-21.

9. Kudo M, Ikeda M, Takayama T, et al. Safety and efficacy of sorafenib in Japanese patients with hepatocellular carcinoma in clinical practice: a subgroup analysis of GIDEON. J Gastroenterol. 2016. doi:10.1007/s00535-016-1204-2.

10. Yoshida H, Shiratori Y, Kudo M, et al. Effect of vitamin K2 on the recurrence of hepatocellular carcinoma. Hepatology. 2011;54:532-40.

11. Yang SL, Liu LP, Sun YF, et al. Distinguished prognosis after hepatectomy of HBV-related hepatocellular carcinoma with or without cirrhosis: a long-term follow-up analysis. J Gastroenterol. 2016;51:722-32.

12. Llovet JM, Bru C, Bruix J, et al. Prognosis of hepatocellular carcinoma: the BCLC staging classification. Semin Liver Dis. 1999;19:329-38.

13. Takayasu K, Arii S, Ikai I, et al. Prospective cohort study of transarterial chemoembolization for unresectable hepatocellular carcinoma in 8510 patients. Gastroenterology. 2006;131:461-9.

14. Asahina Y, Tsuchiya K, Nishimura T, et al. alpha-fetoprotein levels after interferon therapy and risk of hepatocarcinogenesis in chronic hepatitis C. Hepatology. 2013;58:1253-62.

15. Ogawa E, Furusyo N, Kajiwara E, et al. Efficacy of pegylated interferon alpha- $2 b$ and ribavirin treatment on the risk of hepatocellular carcinoma in patients with chronic hepatitis $\mathrm{C}$ : a prospective, multicenter study. J Hepatol. 2013;58:495-501.

16. You JS, Jones PA. Cancer genetics and epigenetics: two sides of the same coin? Cancer Cell. 2012;22:9-20.

17. Baylin SB, Jones PA. A decade of exploring the cancer epigenome-biological and translational implications. Nat Rev Cancer. 2011;11:726-34.

18. Chiba T, Marusawa H, Ushijima T. Inflammation-associated cancer development in digestive organs: mechanisms and roles for genetic and epigenetic modulation. Gastroenterology. 2012;143:550-63. 
19. Totoki Y, Tatsuno K, Yamamoto S, et al. High-resolution characterization of a hepatocellular carcinoma genome. Nat Genet. 2011;43:464-9.

20. Fujimoto A, Totoki Y, Abe T, et al. Whole-genome sequencing of liver cancers identifies etiological influences on mutation patterns and recurrent mutations in chromatin regulators. Nat Genet. 2012;44:760-4.

21. Guichard C, Amaddeo G, Imbeaud S, et al. Integrated analysis of somatic mutations and focal copy-number changes identifies key genes and pathways in hepatocellular carcinoma. Nat Genet. 2012;44:694-8.

22. Huang J, Deng Q, Wang Q, et al. Exome sequencing of hepatitis B virus-associated hepatocellular carcinoma. Nat Genet. 2012;44:1117-21.

23. Chen Y, Wang L, Xu H, et al. Exome capture sequencing reveals new insights into hepatitis $\mathrm{B}$ virus-induced hepatocellular carcinoma at the early stage of tumorigenesis. Oncol Rep. 2013;30:1906-12.

24. Shibata T, Aburatani H. Exploration of liver cancer genomes. Nat Rev Gastroenterol Hepatol. 2014;11:340-9.

25. Totoki Y, Tatsuno K, Covington KR, et al. Trans-ancestry mutational landscape of hepatocellular carcinoma genomes. Nat Genet. 2014;46:1267-73.

26. Cornella H, Alsinet C, Sayols S, et al. Unique genomic profile of fibrolamellar hepatocellular carcinoma. Gastroenterology. 2015;148:806-18.

27. Fujimoto A, Furuta M, Shiraishi Y, et al. Whole-genome mutational landscape of liver cancers displaying biliary phenotype reveals hepatitis impact and molecular diversity. Nat Commun. 2015;6:6120.

28. Schulze K, Imbeaud S, Letouze E, et al. Exome sequencing of hepatocellular carcinomas identifies new mutational signatures and potential therapeutic targets. Nat Genet. 2015;47:505-11.

29. Schulze K, Nault JC, Villanueva A. Genetic profiling of hepatocellular carcinoma using next-generation sequencing. J Hepatol. 2016. doi:10.1016/j.jhep.2016.05.035.

30. Kim SK, Ueda Y, Hatano E, et al. TERT promoter mutations and chromosome $8 p$ loss are characteristic of nonalcoholic fatty liver disease-related hepatocellular carcinoma. Int $\mathrm{J}$ Cancer. 2016;139:2512-8.

31. World Health Organization. Fact sheets: Hepatitis C. http://www. who.int/mediacentre/factsheets/fs 164/en/. Accessed 1 Sept 2016.

32. Alberti A, Chemello L, Benvegnu L. Natural history of hepatitis C. J Hepatol. 1999;31:17-24.

33. Ikeda K, Marusawa H, Osaki Y, et al. Antibody to hepatitis B core antigen and risk for hepatitis C-related hepatocellular carcinoma: a prospective study. Ann Intern Med. 2007;146:649-56.

34. Fattovich G, Pantalena M, Zagni I, et al. Effect of hepatitis B and $\mathrm{C}$ virus infections on the natural history of compensated cirrhosis: a cohort study of 297 patients. Am J Gastroenterol. 2002;97:2886-95.

35. Ikeda K, Saitoh S, Koida I, et al. A multivariate analysis of risk factors for hepatocellular carcinogenesis: a prospective observation of 795 patients with viral and alcoholic cirrhosis. Hepatology. 1993;18:47-53.

36. Ikeda K, Arase Y, Saitoh S, et al. Prediction model of hepatocarcinogenesis for patients with hepatitis $\mathrm{C}$ virus-related cirrhosis. Validation with internal and external cohorts. J Hepatol. 2006;44:1089-97.

37. Lee MH, Yang HI, Lu SN, et al. Hepatitis C virus seromarkers and subsequent risk of hepatocellular carcinoma: long-term predictors from a community-based cohort study. J Clin Oncol. 2010;28:4587-93.

38. Osaki Y, Ueda Y, Marusawa H, et al. Decrease in alpha-fetoprotein levels predicts reduced incidence of hepatocellular carcinoma in patients with hepatitis $\mathrm{C}$ virus infection receiving interferon therapy: a single center study. J Gastroenterol. 2012;47:444-51.

39. Liu CH, Liu CJ, Lin CL, et al. Pegylated interferon-alpha-2a plus ribavirin for treatment-naive Asian patients with hepatitis $\mathrm{C}$ virus genotype 1 infection: a multicenter, randomized controlled trial. Clin Infect Dis. 2008;47:1260-9.

40. Forns X, Lawitz E, Zeuzem S, et al. Simeprevir with peginterferon and ribavirin leads to high rates of SVR in patients with HCV genotype 1 who relapsed after previous therapy: a phase 3 trial. Gastroenterology. 2014;146:1669-79.

41. Hayashi N, Izumi N, Kumada H, et al. Simeprevir with peginterferon/ribavirin for treatment-naive hepatitis $\mathrm{C}$ genotype 1 patients in Japan: CONCERTO-1, a phase III trial. J Hepatol. 2014;61:219-27.

42. Izumi N, Hayashi N, Kumada H, et al. Once-daily simeprevir with peginterferon and ribavirin for treatment-experienced $\mathrm{HCV}$ genotype 1-infected patients in Japan: the CONCERTO-2 and CONCERTO-3 studies. J Gastroenterol. 2014;49:941-53.

43. Manns M, Marcellin P, Poordad F, et al. Simeprevir with pegylated interferon alfa $2 \mathrm{a}$ or $2 \mathrm{~b}$ plus ribavirin in treatmentnaive patients with chronic hepatitis $\mathrm{C}$ virus genotype 1 infection (QUEST-2): a randomised, double-blind, placebo-controlled phase 3 trial. Lancet. 2014;384:414-26.

44. Manns M, Pol S, Jacobson IM, et al. All-oral daclatasvir plus asunaprevir for hepatitis $\mathrm{C}$ virus genotype $1 \mathrm{~b}$ : a multinational, phase 3, multicohort study. Lancet. 2014;384:1597-605.

45. Kumada H, Suzuki Y, Ikeda K, et al. Daclatasvir plus asunaprevir for chronic HCV genotype $1 \mathrm{~b}$ infection. Hepatology. 2014;59:2083-91.

46. Afdhal N, Reddy KR, Nelson DR, et al. Ledipasvir and sofosbuvir for previously treated HCV genotype 1 infection. N Engl J Med. 2014;370:1483-93.

47. Afdhal N, Zeuzem S, Kwo P, et al. Ledipasvir and sofosbuvir for untreated HCV genotype 1 infection. $N$ Engl $J$ Med. 2014;370:1889-98.

48. Kumada H, Chayama K, Rodrigues L Jr, et al. Randomized phase 3 trial of ombitasvir/paritaprevir/ritonavir for hepatitis $\mathrm{C}$ virus genotype $1 \mathrm{~b}$-infected Japanese patients with or without cirrhosis. Hepatology. 2015;62:1037-46.

49. Yoshida H, Shiratori Y, Moriyama M, et al. Interferon therapy reduces the risk for hepatocellular carcinoma: national surveillance program of cirrhotic and noncirrhotic patients with chronic hepatitis C in Japan. IHIT Study Group. Ann Intern Med. 1999;131:174-81.

50. George SL, Bacon BR, Brunt EM, et al. Clinical, virologic, histologic, and biochemical outcomes after successful HCV therapy: a 5-year follow-up of 150 patients. Hepatology. 2009;49:729-38.

51. Cardoso AC, Moucari R, Figueiredo-Mendes C, et al. Impact of peginterferon and ribavirin therapy on hepatocellular carcinoma: incidence and survival in hepatitis $\mathrm{C}$ patients with advanced fibrosis. J Hepatol. 2010;52:652-7.

52. Hiramatsu N, Oze T, Takehara T. Suppression of hepatocellular carcinoma development in hepatitis $\mathrm{C}$ patients given interferonbased antiviral therapy. Hepatol Res. 2015;45:152-61.

53. El-Serag HB, Kanwal F, Richardson P, et al. Risk of Hepatocellular Carcinoma after Sustained Virologic Response in Veterans with HCV-infection. Hepatology. 2016;64:130-7.

54. Reig M, Marino Z, Perello C, et al. Unexpected early tumor recurrence in patients with hepatitis $\mathrm{C}$ virus -related hepatocellular carcinoma undergoing interferon-free therapy: a note of caution. J Hepatol. 2016;65:719-26.

55. Toyoda H, Kumada T, Tada T. Changes in patient backgrounds may increase the incidence of HCC after SVR in the era of IFNfree therapy for HCV. Hepatology. 2016. doi:10.1002/hep. 28632. 
56. World Health Organization. Fact Sheets: Hepatitis B. http:// www.who.int/mediacentre/factsheets/fs204/en/. Accessed 1 Sept 2016.

57. Levrero M, Zucman-Rossi J. Mechanisms of HBV-induced hepatocellular carcinoma. J Hepatol. 2016;64:S84-101.

58. Fattovich G, Stroffolini T, Zagni I, et al. Hepatocellular carcinoma in cirrhosis: incidence and risk factors. Gastroenterology. 2004;127:S35-50.

59. Chen JD, Yang HI, Iloeje UH, et al. Carriers of inactive hepatitis $\mathrm{B}$ virus are still at risk for hepatocellular carcinoma and liverrelated death. Gastroenterology. 2010;138:1747-54.

60. Chen CJ, Yang HI, Su J, et al. Risk of hepatocellular carcinoma across a biological gradient of serum hepatitis B virus DNA level. JAMA. 2006;295:65-73.

61. Dienstag JL, Perrillo RP, Schiff ER, et al. A preliminary trial of lamivudine for chronic hepatitis B infection. N Engl J Med. 1995;333:1657-61.

62. Nevens F, Main J, Honkoop P, et al. Lamivudine therapy for chronic hepatitis B: a six-month randomized dose-ranging study. Gastroenterology. 1997;113:1258-63.

63. Marcellin P, Chang TT, Lim SG, et al. Adefovir dipivoxil for the treatment of hepatitis B e antigen-positive chronic hepatitis B. N Engl J Med. 2003;348:808-16.

64. Liaw YF, Sung JJ, Chow WC, et al. Lamivudine for patients with chronic hepatitis B and advanced liver disease. N Engl J Med. 2004;351:1521-31.

65. Lai CL, Shouval D, Lok AS, et al. Entecavir versus lamivudine for patients with $\mathrm{HBeAg}$-negative chronic hepatitis B. N Engl J Med. 2006;354:1011-20.

66. Marcellin P, Heathcote EJ, Buti M, et al. Tenofovir disoproxil fumarate versus adefovir dipivoxil for chronic hepatitis B. N Engl J Med. 2008;359:2442-55.

67. Lin SM, Sheen IS, Chien RN, et al. Long-term beneficial effect of interferon therapy in patients with chronic hepatitis B virus infection. Hepatology. 1999;29:971-5.

68. Wu CY, Chen YJ, Ho HJ, et al. Association between nucleoside analogues and risk of hepatitis B virus-related hepatocellular carcinoma recurrence following liver resection. JAMA. 2012;308:1906-14.

69. Marusawa H, Imoto S, Ueda Y, et al. Reactivation of latently infected hepatitis B virus in a leukemia patient with antibodies to hepatitis B core antigen. J Gastroenterol. 2001;36:633-6.

70. Inuzuka T, Ueda Y, Morimura H, et al. Reactivation from occult HBV carrier status is characterized by low genetic heterogeneity with the wild-type or G1896A variant prevalence. J Hepatol. 2014;61:492-501.

71. Marusawa H, Uemoto S, Hijikata M, et al. Latent hepatitis B virus infection in healthy individuals with antibodies to hepatitis B core antigen. Hepatology. 2000;31:488-95.

72. Hudson TJ, Anderson W, Artez A, et al. International network of cancer genome projects. Nature. 2010;464:993-8.

73. Fujimoto A, Furuta M, Totoki Y, et al. Whole-genome mutational landscape and characterization of noncoding and structural mutations in liver cancer. Nat Genet. 2016;48:500-9.

74. Kawai-Kitahata F, Asahina Y, Tanaka S, et al. Comprehensive analyses of mutations and hepatitis B virus integration in hepatocellular carcinoma with clinicopathological features. J Gastroenterol. 2016;51:473-86.

75. Vogelstein B, Papadopoulos N, Velculescu VE, et al. Cancer genome landscapes. Science. 2013;339:1546-58.

76. Nault JC, Mallet M, Pilati C, et al. High frequency of telomerase reverse-transcriptase promoter somatic mutations in hepatocellular carcinoma and preneoplastic lesions. Nat Commun. 2013;4:2218.
77. Zucman-Rossi J, Villanueva A, Nault JC, et al. Genetic Landscape and Biomarkers of Hepatocellular Carcinoma. Gastroenterology. 2015;149:1226-39.

78. Greenman C, Stephens P, Smith R, et al. Patterns of somatic mutation in human cancer genomes. Nature. 2007;446:153-8.

79. Kan Z, Zheng H, Liu X, et al. Whole-genome sequencing identifies recurrent mutations in hepatocellular carcinoma. Genome Res. 2013;23:1422-33.

80. Ahn SM, Jang SJ, Shim JH, et al. Genomic portrait of resectable hepatocellular carcinomas: implications of RB1 and FGF19 aberrations for patient stratification. Hepatology. 2014;60:1972-82.

81. Arao T, Ueshima K, Matsumoto K, et al. FGF3/FGF4 amplification and multiple lung metastases in responders to sorafenib in hepatocellular carcinoma. Hepatology. 2013;57:1407-15.

82. Llovet JM. Focal gains of VEGFA: candidate predictors of sorafenib response in hepatocellular carcinoma. Cancer Cell. 2014;25:560-2.

83. Garcia M, de The H, Tiollais P, et al. A hepatitis B virus pre-Sretinoic acid receptor beta chimera transforms erythrocytic progenitor cells in vitro. PNAS. 1993;90:89-93.

84. Wang J, Zindy F, Chenivesse X, et al. Modification of cyclin A expression by hepatitis B virus DNA integration in a hepatocellular carcinoma. Oncogene. 1992;7:1653-6.

85. Sung WK, Zheng H, Li S, et al. Genome-wide survey of recurrent $\mathrm{HBV}$ integration in hepatocellular carcinoma. Nat Genet. 2012;44:765-9.

86. Lau CC, Sun T, Ching AK, et al. Viral-human chimeric transcript predisposes risk to liver cancer development and progression. Cancer Cell. 2014;25:335-49.

87. Nault JC, Datta S, Imbeaud S, et al. Recurrent AAV2-related insertional mutagenesis in human hepatocellular carcinomas. Nat Genet. 2015;47:1187-93.

88. Atchison RW, Casto BC, Hammon WM. Adenovirus-associated defective virus particles. Science. 1965;149:754-6.

89. Russell DW, Grompe M. Adeno-associated virus finds its disease. Nat Genet. 2015;47:1104-5.

90. Nishida N, Nagasaka T, Nishimura T, et al. Aberrant methylation of multiple tumor suppressor genes in aging liver, chronic hepatitis, and hepatocellular carcinoma. Hepatology. 2008;47:908-18.

91. Pogribny IP, Rusyn I. Role of epigenetic aberrations in the development and progression of human hepatocellular carcinoma. Cancer Lett. 2014;342:223-30.

92. Shen J, Wang S, Zhang YJ, et al. Genome-wide DNA methylation profiles in hepatocellular carcinoma. Hepatology. 2012;55:1799-808.

93. Tao R, Li J, Xin J, et al. Methylation profile of single hepatocytes derived from hepatitis B virus-related hepatocellular carcinoma. PLoS One. 2011;6:e19862.

94. Kanai Y, Ushijima S, Nakanishi Y, et al. Mutation of the DNA methyltransferase (DNMT) 1 gene in human colorectal cancers. Cancer Lett. 2003;192:75-82.

95. Lujambio A, Lowe SW. The microcosmos of cancer. Nature. 2012;482:347-55.

96. Shen J, Wang S, Zhang YJ, et al. Genome-wide aberrant DNA methylation of microRNA host genes in hepatocellular carcinoma. Epigenetics. 2012;7:1230-7.

97. Liu H, Liu Y, Liu W, et al. EZH2-mediated loss of miR-622 determines CXCR4 activation in hepatocellular carcinoma. Nat Commun. 2015;6:8494.

98. Liu Z, Dou C, Yao B, et al. Methylation-mediated repression of microRNA-129-2 suppresses cell aggressiveness by inhibiting high mobility group box 1 in human hepatocellular carcinoma. Oncotarget. 2016. doi:10.18632/oncotarget.9377. 
99. Wu WR, Sun H, Zhang R, et al. Methylation-associated silencing of miR-200b facilitates human hepatocellular carcinoma progression by directly targeting BMI1. Oncotarget. 2016;7:18684-93.

100. Varley KE, Gertz J, Bowling KM, et al. Dynamic DNA methylation across diverse human cell lines and tissues. Genome Res. 2013;23:555-67.

101. Xiao-Jie L, Hui-Ying X, Qi X, et al. LINE-1 in cancer: multifaceted functions and potential clinical implications. Genet Med. 2016;18:431-9.

102. Lee HS, Kim BH, Cho NY, et al. Prognostic implications of and relationship between $\mathrm{CpG}$ island hypermethylation and repetitive DNA hypomethylation in hepatocellular carcinoma. Clin Cancer Res. 2009;15:812-20.

103. Feng Q, Stern JE, Hawes SE, et al. DNA methylation changes in normal liver tissues and hepatocellular carcinoma with different viral infection. Exp Mol Pathol. 2010;88:287-92.

104. Neumann O, Kesselmeier M, Geffers R, et al. Methylome analysis and integrative profiling of human HCCs identify novel protumorigenic factors. Hepatology. 2012;56:1817-27.

105. Deng YB, Nagae G, Midorikawa Y, et al. Identification of genes preferentially methylated in hepatitis $\mathrm{C}$ virus-related hepatocellular carcinoma. Cancer Sci. 2010;101:1501-10.

106. Shimizu T, Marusawa H, Endo Y, et al. Inflammation-mediated genomic instability: roles of activation-induced cytidine deaminase in carcinogenesis. Cancer Sci. 2012;103:1201-6.

107. Hussain SP, Hofseth LJ, Harris CC. Radical causes of cancer. Nat Rev Cancer. 2003;3:276-85.

108. Shibutani S, Takeshita M, Grollman AP. Insertion of specific bases during DNA synthesis past the oxidation-damaged base 8-oxodG. Nature. 1991;349:431-4.

109. Wink DA, Kasprzak KS, Maragos CM, et al. DNA deaminating ability and genotoxicity of nitric oxide and its progenitors. Science. 1991;254:1001-3.

110. Jaeschke H, Farhood A. Neutrophil and Kupffer cell-induced oxidant stress and ischemia-reperfusion injury in rat liver. Am J Physiol. 1991;260:355-62.

111. Wynalda MA, Hauer MJ, Wienkers LC. Oxidation of the novel oxazolidinone antibiotic linezolid in human liver microsomes. Drug Metab Dispos. 2000;28:1014-7.

112. Poirier Y, Antonenkov VD, Glumoff T, et al. Peroxisomal betaoxidation-a metabolic pathway with multiple functions. Biochim Biophys Acta. 2006;1763:1413-26.

113. de Mochel NS, Seronello S, Wang SH, et al. Hepatocyte $\mathrm{NAD}(\mathrm{P}) \mathrm{H}$ oxidases as an endogenous source of reactive oxygen species during hepatitis $\mathrm{C}$ virus infection. Hepatology. 2010;52:47-59.

114. Jungst C, Cheng B, Gehrke R, et al. Oxidative damage is increased in human liver tissue adjacent to hepatocellular carcinoma. Hepatology. 2004;39:1663-72.

115. Dionisio N, Garcia-Mediavilla MV, Sanchez-Campos S, et al. Hepatitis C virus NS5A and core proteins induce oxidative stress-mediated calcium signalling alterations in hepatocytes. J Hepatol. 2009;50:872-82.

116. Machida K, Cheng KT, Sung VM, et al. Hepatitis C virus infection activates the immunologic (type II) isoform of nitric oxide synthase and thereby enhances DNA damage and mutations of cellular genes. J Virol. 2004;78:8835-43.

117. Gong G, Waris G, Tanveer R, et al. Human hepatitis C virus NS5A protein alters intracellular calcium levels, induces oxidative stress, and activates STAT-3 and NF-kappa B. PNAS. 2001;98:9599-604.

118. Waris G, Turkson J, Hassanein T, et al. Hepatitis C virus (HCV) constitutively activates STAT-3 via oxidative stress: role of STAT-3 in HCV replication. J Virol. 2005;79:1569-80.
119. Machida K, McNamara G, Cheng KT, et al. Hepatitis C virus inhibits DNA damage repair through reactive oxygen and nitrogen species and by interfering with the ATM-NBS1/Mre11/ Rad50 DNA repair pathway in monocytes and hepatocytes. J Immunol. 2010;185:6985-98.

120. Matsumoto Y, Marusawa H, Kinoshita K, et al. Helicobacter pylori infection triggers aberrant expression of activation-induced cytidine deaminase in gastric epithelium. Nat Med. 2007;13:470-6.

121. Shimizu T, Marusawa H, Matsumoto $Y$, et al. Accumulation of somatic mutations in TP53 in gastric epithelium with Helicobacter pylori infection. Gastroenterology. 2014;147:407-17.

122. Takai A, Marusawa H, Minaki Y, et al. Targeting activationinduced cytidine deaminase prevents colon cancer development despite persistent colonic inflammation. Oncogene. 2012;31:1733-42.

123. Muramatsu M, Sankaranand VS, Anant S, et al. Specific expression of activation-induced cytidine deaminase (AID), a novel member of the RNA-editing deaminase family in germinal center B cells. J Biol Chem. 1999;274:18470-6.

124. Muramatsu M, Kinoshita K, Fagarasan S, et al. Class switch recombination and hypermutation require activation-induced cytidine deaminase (AID), a potential RNA editing enzyme. Cell. 2000;102:553-63.

125. Marusawa H, Takai A, Chiba T. Role of activation-induced cytidine deaminase in inflammation-associated cancer development. Adv Immunol. 2011;111:109-41.

126. Endo Y, Marusawa H, Kinoshita K, et al. Expression of activation-induced cytidine deaminase in human hepatocytes via NF-kappaB signaling. Oncogene. 2007;26:5587-95.

127. Kou T, Marusawa H, Kinoshita K, et al. Expression of activation-induced cytidine deaminase in human hepatocytes during hepatocarcinogenesis. Int J Cancer. 2007;120:469-76.

128. Matsumoto T, Shimizu T, Nishijima N, et al. Hepatic inflammation facilitates transcription-associated mutagenesis via AID activity and enhances liver tumorigenesis. Carcinogenesis. 2015;36:904-13.

129. Kim SK, Nasu A, Komori J, et al. A model of liver carcinogenesis originating from hepatic progenitor cells with accumulation of genetic alterations. Int J Cancer. 2014;134:1067-76.

130. Lange SS, Takata K, Wood RD. DNA polymerases and cancer. Nat Rev Cancer. 2011;11:96-110.

131. Liu M, Duke JL, Richter DJ, et al. Two levels of protection for the B cell genome during somatic hypermutation. Nature. 2008;451:841-5.

132. Imai $\mathrm{K}$, Yamamoto $\mathrm{H}$. Carcinogenesis and microsatellite instability: the interrelationship between genetics and epigenetics. Carcinogenesis. 2008;29:673-80.

133. Rayner E, van Gool IC, Palles C, et al. A panoply of errors: polymerase proofreading domain mutations in cancer. Nat Rev Cancer. 2016;16:71-81.

134. Eso Y, Takai A, Matsumoto T, et al. MSH2 Dysregulation is triggered by proinflammatory cytokine stimulation and is associated with liver cancer development. Cancer Res. 2016;76:4383-93.

135. Machida K, Cheng KT, Sung VM, et al. Hepatitis C virus induces a mutator phenotype: enhanced mutations of immunoglobulin and protooncogenes. PNAS. 2004;101:4262-7.

136. Forgues M, Difilippantonio MJ, Linke SP, et al. Involvement of $\mathrm{Crm} 1$ in hepatitis B virus $\mathrm{X}$ protein-induced aberrant centriole replication and abnormal mitotic spindles. Mol Cell Biol. 2003;23:5282-92.

137. Bouchard MJ, Navas-Martin S. Hepatitis B and C virus hepatocarcinogenesis: lessons learned and future challenges. Cancer Lett. 2011;305:123-43. 
138. Ikeda A, Shimizu T, Matsumoto Y, et al. Leptin receptor somatic mutations are frequent in $\mathrm{HCV}$-infected cirrhotic liver and associated with hepatocellular carcinoma. Gastroenterology. 2014;146:222-32.

139. Nault JC, Calderaro J, Di Tommaso L, et al. Telomerase reverse transcriptase promoter mutation is an early somatic genetic alteration in the transformation of premalignant nodules in hepatocellular carcinoma on cirrhosis. Hepatology. 2014;60:1983-92.

140. Nishida N, Kudo M, Nagasaka T, et al. Characteristic patterns of altered DNA methylation predict emergence of human hepatocellular carcinoma. Hepatology. 2012;56:994-1003.

141. Lee S, Lee HJ, Kim JH, et al. Aberrant CpG island hypermethylation along multistep hepatocarcinogenesis. Am J Pathol. 2003;163:1371-8.

142. Um TH, Kim H, Oh BK, et al. Aberrant CpG island hypermethylation in dysplastic nodules and early $\mathrm{HCC}$ of hepatitis $\mathrm{B}$ virus-related human multistep hepatocarcinogenesis. J Hepatol. 2011;54:939-47.

143. Calvisi DF, Ladu S, Gorden A, et al. Mechanistic and prognostic significance of aberrant methylation in the molecular pathogenesis of human hepatocellular carcinoma. J Clin Invest. 2007;117:2713-22.

144. Okamoto Y, Shinjo K, Shimizu Y, et al. Hepatitis virus infection affects DNA methylation in mice with humanized livers. Gastroenterology. 2014;146:562-72.

145. Nishida N, Arizumi T, Takita M, et al. Reactive oxygen species induce epigenetic instability through the formation of 8-hydroxydeoxyguanosine in human hepatocarcinogenesis. Dig Dis. 2013;31:459-66.

146. Park IY, Sohn BH, Yu E, et al. Aberrant epigenetic modifications in hepatocarcinogenesis induced by hepatitis $\mathrm{B}$ virus $\mathrm{X}$ protein. Gastroenterology. 2007;132:1476-94.

147. Gao W, Kondo Y, Shen L, et al. Variable DNA methylation patterns associated with progression of disease in hepatocellular carcinomas. Carcinogenesis. 2008;29:1901-10.

148. Tokushige K, Hyogo H, Nakajima T, et al. Hepatocellular carcinoma in Japanese patients with nonalcoholic fatty liver disease and alcoholic liver disease: multicenter survey. J Gastroenterol. 2016;51:586-96. 